\title{
Magnetic Resonance Imaging
}

\author{
Charles L. Epstein* and Felix W. Wehrli ${ }^{\dagger}$
}

June 3, 2005

\section{Introduction}

Nuclear magnetic resonance (NMR) is a subtle quantum mechanical phenomenon that, through magnetic resonance imaging (MRI), has played a major role in the revolution in medical imaging over the last 30 years. Before being conceived for use in imaging, NMR was employed by chemists to do spectroscopy, and remains a very important technique for determining the structure of complex chemical compounds like proteins. In this article we explain how NMR is used to create an image of a 3-dimensional object. Scant attention is paid to both NMR spectroscopy, and the quantum description of NMR. Those seeking a more complete introduction to these subjects should consult [5], as well as the monographs of Abragam, [1], or Ernst, Bodenhausen and Wokaun, [12], for spectroscopy, and that of Callaghan, [4], for imaging. All three books consider the quantum mechanical description of these phenomena. Comprehensive discussions of MRI can be found in $[3,13]$, and an historical appreciation of the development of MRI is given in [19].

\section{The Bloch Equation}

We begin with the Bloch phenomenological equation, which provides a model for the interactions between applied magnetic fields and the nuclear spins in the objects under consideration. This is a macroscopic averaged model that describes the interaction of aggregates of spins, called isochromats, with applied magnetic fields. An isochromat is a collection of "like" spins, which is spatially large on the atomic scale, but very small on the scale of the variations present in the applied magnetic fields. Spins are alike if they belong to the same species and are in the same chemical environment. There may be several different classes of spins, but, in this article, it is assumed that they are non-interacting and so it suffices to consider each separately. Heretofore we

\footnotetext{
*Address: Department of Mathematics, 209 S. 33rd Street, Philadelphia, PA, 19104-6395 USA. E-mail: cle@math.upenn.edu

†Address: Laboratory for Structural NMR Imaging, Department of Radiology, 1 Founders, University of Pennsylvania Medical Center, 3400 Spruce Street, Philadelphia, PA 19104, USA Email: wehrlif@uphs.upenn.edu
} 
suppose that there is a single class of like spins. The distribution of isochromats for these spins is described macroscopically by the spin density function, which we denote by $\rho(x, y, z)$. In most medical applications, one is imaging the distribution of spins arising from hydrogen protons in water molecules.

The state of the isochromat at spatial location $(x, y, z)$ is given by a 3 -vector:

$$
\boldsymbol{M}(x, y, z)=\left(m_{1}(x, y, z), m_{2}(x, y, z), m_{3}(x, y, z)\right),
$$

which is interpreted as the magnetic moment-per-unit-volume. It is an ensemble mean of the quantum dipoles caused by the spins within the isochromat. In most applications of NMR to imaging, the applied magnetic field is described as the sum of a large, time independent field, $\boldsymbol{B}_{0}(x, y, z)$, and smaller time dependent fields, $\boldsymbol{B}^{\prime}(x, y, z ; t)$. In the presence of a static field, thermal fluctuations cause the nuclear spins to slightly prefer an orientation aligned with the field. Using the Boltzmann distribution, one obtains that, at high temperatures, the nuclear paramagnetic susceptibility of protons is given by

$$
\chi \approx \frac{\mu_{0} \hbar^{2} \gamma^{2} N}{4 k_{B} T}
$$

here $\mu_{0}$ is the vacuum permeability, $\hbar$ is Planck's constant, $N$ is the number of protons per unit volume, $k_{B}$ is Boltzmann's constant, and $T$ is the absolute temperature, see [15]. The constant $\gamma$ is called the gyromagnetic (or magnetogyric) ratio. For a proton

$$
\gamma \approx 2 \pi \times 42.5764 \times 10^{6} \frac{\mathrm{rad}}{\mathrm{s} \cdot \mathrm{Tesla}}
$$

For water molecules at room temperature $\chi \approx 3.6 \times 10^{-9}$.

If the sample is held stationary in the $\boldsymbol{B}_{0}$-field for a sufficiently long time, then the spins become polarized and a bulk magnetic moment appears; this is called the equilibrium magnetization:

$$
\boldsymbol{M}_{0}(x, y, z)=\chi \rho(x, y, z) \boldsymbol{B}_{0}(x, y, z) .
$$

The Bloch equation describes the evolution of $\boldsymbol{M}$ under the influence of the applied field $\boldsymbol{B}=\boldsymbol{B}_{0}+\boldsymbol{B}^{\prime}$ :

$$
\begin{aligned}
\frac{d \boldsymbol{M}(x, y, z ; t)}{d t}= & \gamma \boldsymbol{M}(x, y, z ; t) \times \boldsymbol{B}(x, y, z ; t)-\frac{1}{T_{2}} \boldsymbol{M}^{\perp}(x, y, z ; t)+ \\
& \frac{1}{T_{1}}\left(\boldsymbol{M}_{0}(x, y, z)-\boldsymbol{M}^{\|}(x, y, z ; t)\right) .
\end{aligned}
$$

Here $\times$ is the vector cross product, $\boldsymbol{M}^{\perp}(x, y, z ; t)$ is the component of $\boldsymbol{M}(x, y, z ; t)$, perpendicular to $\boldsymbol{B}_{0}(x, y, z)$, (called the transverse component), and $\boldsymbol{M}^{\|}$is the component of $\boldsymbol{M}$, parallel to $\boldsymbol{B}_{0}$, (called the longitudinal component). For hydrogen protons in other molecules, the gyromagnetic ratio is expressed in the form $(1-\sigma) \gamma$. The coefficient $\sigma$ is called the nuclear shielding; it is typically between $-10^{-4}$ and $+10^{-4}$. The difference in the nuclear shielding causes a shift in the resonance frequency by $\gamma \sigma$.

The second and third terms in equation (4) are relaxation terms. They provide a phenomenological model for the averaged interactions of the spins with one another 
and their environment. The coefficient $1 / T_{1}(x, y, z)$ is the spin lattice relaxation rate; it describes the rate at which the magnetization returns to equilibrium. The coefficient $1 / T_{2}(x, y, z)$ is the spin-spin relaxation rate; it describes the rate at which the transverse components of $\boldsymbol{M}$ decay. The physical processes causing these relaxation phenomena are different and so are the rates themselves, with $T_{2}$ less than $T_{1}$. The relaxation rates largely depend on the localized thermal fluctuations of the molecules and provide a useful contrast mechanism in MR imaging. Spin-spin relaxation occurs very rapidly in solids $(<1 \mathrm{~ms})$ and therefore we usually assume that we are imaging liquid-like materials such as water protons in soft mammalian tissues. In this case $T_{2}$ takes values in the $40 \mathrm{~ms}-4 \mathrm{~s}$ range. Notice that this model does not include any explicit interaction between isochromats at different spatial locations. A variety of such interactions exist, but, at least in liquid like materials, they lead only to small corrections in the Bloch equation model. A derivation of the Bloch equation from the Schrödinger equation can be found in $[1,18]$. For coupled systems the Bloch equation formalism breaks down and a full quantum-mechanical treatment, is necessary see [5, 12].

Much of the analysis in NMR imaging amounts to understanding the behavior of solutions to equation (4) with different choices of $\boldsymbol{B}$. We now consider some important special cases. The simplest case occurs if $\boldsymbol{B}$ has no time dependent component, then this equation predicts that the sample becomes polarized with the transverse part of $\boldsymbol{M}$ decaying as $e^{-\frac{t}{T_{2}}}$, and the longitudinal component approaching the equilibrium magnetization, $\boldsymbol{M}_{0}$, as $1-e^{-\frac{t}{T_{1}}}$. To simplify the subsequent discussion we assume that the $\boldsymbol{B}_{0}$-field is homogeneous with $\boldsymbol{B}_{0}=\left(0,0, b_{0}\right)$. If $\boldsymbol{B}=\boldsymbol{B}_{0}$ and we omit the relaxation terms (set $T_{1}=T_{2}=\infty$ in (4)), then an initial magnetization $\boldsymbol{M}(x, y, z ; 0)$ simply precesses about $\boldsymbol{B}_{0}$ at angular frequency $\omega_{0}=\gamma b_{0}: \boldsymbol{M}(x, y, z ; t)=U(t) \boldsymbol{M}(x, y, z ; 0)$ with

$$
U(t)=\left[\begin{array}{ccc}
\cos \omega_{0} t & -\sin \omega_{0} t & 0 \\
\sin \omega_{0} t & \cos \omega_{0} t & 0 \\
0 & 0 & 1
\end{array}\right] .
$$

The frequency $\omega_{0}$ is called the Larmor frequency; this precession of $\boldsymbol{M}$ about the axis of $\boldsymbol{B}_{0}$ is the resonance phenomenon referred to as "nuclear magnetic resonance" (NMR). In typical medical imaging systems $b_{0}$ is between 1 and 3 Tesla; the corresponding resonance frequency is between 40 and 120 megahertz.

Typically the field $\boldsymbol{B}$ takes the form

$$
\boldsymbol{B}=\boldsymbol{B}_{0}+\widetilde{\boldsymbol{G}}+\boldsymbol{B}_{1},
$$

where $\widetilde{\boldsymbol{G}}$ is a gradient field and $\boldsymbol{B}_{1}$ is an $R F$-field. Usually the gradient fields are "piecewise time independent" fields, small relative to $\boldsymbol{B}_{0}$. By piecewise time independent field we mean a collection of static fields that, in the course of the experiment, are turned on and off. The $\boldsymbol{B}_{1}$ component is a time dependent radio frequency field, nominally at right angles to $\boldsymbol{B}_{0}$. It is usually taken to be spatially homogeneous, with time dependence of the form:

$$
\boldsymbol{B}_{1}(t)=U(t)\left(\begin{array}{c}
\alpha(t) \\
\beta(t) \\
0
\end{array}\right)
$$


The functions $\alpha$ and $\beta$ define an envelope that modulates the time harmonic field, $\left[\cos \omega_{0} t, \sin \omega_{0} t, 0\right]$. They are supported in a finite interval $\left[t_{0}, t_{1}\right]$, i.e, the $\boldsymbol{B}_{1}$ field is "turned on" for a finite period of time. The change in the state of the magnetization between $t_{0}$ and $t_{1}$ is called the $R F$-excitation. It may be spatially dependent.

In light of (5) it is convenient to introduce the rotating reference frame. We replace $\boldsymbol{M}$ with $\boldsymbol{m}$ where $\boldsymbol{m}(x, y, z ; t)=U(t)^{-1} \boldsymbol{M}(x, y, z ; t)$. It is a classical result of Larmor, that if $\boldsymbol{M}$ satisfies (4), then $\boldsymbol{m}$ satisfies

$$
\begin{aligned}
\frac{d \boldsymbol{m}(x, y, z ; t)}{d t}= & \gamma \boldsymbol{m}(x, y, z ; t) \times \boldsymbol{B}_{\mathrm{eff}}(x, y, z ; t)-\frac{1}{T_{2}} \boldsymbol{m}^{\perp}(x, y, z ; t)+ \\
& \frac{1}{T_{1}}\left(\boldsymbol{M}_{0}(x, y, z)-\boldsymbol{m}^{\|}(x, y, z ; t)\right),
\end{aligned}
$$

where

$$
\boldsymbol{B}_{\text {eff }}=U(t)^{-1} \boldsymbol{B}-\left(0,0, \frac{\omega_{0}}{\gamma}\right) .
$$

As $\widetilde{\boldsymbol{G}}$ is much smaller than $\boldsymbol{B}$ and quasi-static, it turns out that one can ignore the components of $\tilde{\boldsymbol{G}}$ orthogonal to $\boldsymbol{B}_{0}$. Indeed, in imaging applications one usually assumes that the components of $\tilde{\boldsymbol{G}}$ depend linearly on $(x, y, z)$ with the $\hat{z}$-component given by $\left\langle(x, y, z),\left(g_{1}, g_{2}, g_{3}\right)\right\rangle$. The constant vector $\boldsymbol{G}=\left(g_{1}, g_{2}, g_{3}\right)$ is called the gradient vector. With $\boldsymbol{B}_{0}=\left(0,0, b_{0}\right)$ and $\boldsymbol{B}_{1}$ given by (7), we see that $\boldsymbol{B}_{\text {eff }}$ can be taken to equal $(0,0,\langle(x, y, z), \boldsymbol{G}\rangle)+(\alpha, \beta, 0)$. In the remainder of this article we assume that $\boldsymbol{B}_{\text {eff }}$ takes this form.

If $\boldsymbol{G}=0$ and $\beta \equiv 0$, then the solution operator for Bloch's equation, without relaxation terms, is

$$
V(t)=\left[\begin{array}{ccc}
1 & 0 & 0 \\
0 & \cos \theta(t) & \sin \theta(t) \\
0 & -\sin \theta(t) & \cos \theta(t)
\end{array}\right]
$$

where

$$
\theta(t)=\int_{0}^{t} \alpha(s) d s .
$$

This is simply a rotation about the $x$-axis through the angle $\theta(t)$. If $\boldsymbol{B}_{1} \neq 0$ for $t \in[0, \tau]$, then the magnetization is rotated through the angle $\theta(\tau)$. Thus RF-excitation can be used to move the magnetization out of its equilibrium state. As we shall soon see, this is crucial for obtaining a measurable signal. Nota bene: the equilibrium magnetization is a tiny perturbation of the very large $\boldsymbol{B}_{0}$-field and is therefore in practice not directly measurable. Only the precessional motion of the transverse components of $\boldsymbol{M}$ produces a measurable signal. More general $\boldsymbol{B}_{1}$-fields, i.e. with both $\alpha$ and $\beta$ non-zero, have more complicated effects on the magnetization. In general the angle between $\boldsymbol{M}$ and $\boldsymbol{M}_{0}$ at the conclusion of the RF-excitation is called the flip angle.

If, on the other hand, $\boldsymbol{B}_{1}=0$ and $\boldsymbol{G}_{l}=(0,0, l(x, y, z))$, where $l(\cdot)$ is a function, then $V$ depends on $(x, y, z)$, and is given by

$$
V(x, y, z ; t)=\left[\begin{array}{ccc}
\cos \gamma l(x, y, z) t & -\sin \gamma l(x, y, z) t & 0 \\
\sin \gamma l(x, y, z) t & \cos \gamma l(x, y, z) t & 0 \\
0 & 0 & 1
\end{array}\right]
$$


This is precession about $\boldsymbol{B}_{0}$ at an angular frequency that depends on the local field strength $b_{0}+l(x, y, z)$. If both $\boldsymbol{B}_{1}$ and $\widetilde{\boldsymbol{G}}$ are simultaneously non-zero, then, starting from equilibrium, the solution of the Bloch equation, at the conclusion of the RF-pulse, has a nontrivial spatial dependence. In other words, the flip angle becomes a function of the spatial variables. We return to this in Section 4.

\section{A basic imaging experiment}

With these preliminaries we can describe the basic measurements in magnetic resonance imaging. When exposed to $\boldsymbol{B}_{0}$, the sample becomes polarized at a rate determined by $T_{1}$. Once the sample is polarized, a $\boldsymbol{B}_{1}$-field, of the form given in (7), (with $\beta \equiv 0$ ) is turned on for a finite time $\tau$. This is called an RF-excitation. For the purposes of this discussion we suppose that the time is chosen so that $\theta(\tau)=90^{\circ}$, see equation (10). As $\boldsymbol{B}_{0}$ and $\boldsymbol{B}_{1}$ are spatially homogeneous, the magnetization vectors within the object remain parallel throughout the RF-excitation. At the conclusion of the RF-excitation, $\boldsymbol{M}$ is orthogonal to $\boldsymbol{B}_{0}$.

After the RF is turned off, the vector field $\boldsymbol{M}(x, y, z ; t)$ precesses about $\boldsymbol{B}_{0}$, in phase, with angular velocity $\omega_{0}$. The transverse component of $\boldsymbol{M}$ decays exponentially. If we normalize the time so that $t=0$ corresponds to the conclusion of the RF-pulse, then, in the laboratory frame,

$$
\boldsymbol{M}(x, y, z ; t)=\frac{\chi \omega_{0} \rho(x, y, z)}{\gamma}\left[e^{-\frac{t}{T_{2}}} \cos \omega_{0} t, e^{-\frac{t}{T_{2}}} \sin \omega_{0} t,\left(1-e^{-\frac{t}{T_{1}}}\right)\right] .
$$

Recall Faraday's Law: A changing magnetic field induces an electro-motive force (EMF) in a loop of wire according to the relation

$$
\mathrm{EMF}_{\text {loop }} \propto \frac{d \Phi_{\text {loop }}}{d t} .
$$

Here $\Phi_{\text {loop }}$ denotes the flux of the field through the loop of wire, see [20]. The transverse components of $\boldsymbol{M}$ are a rapidly varying magnetic field, which, according to Faraday's law, induce a current in a loop of wire. In fact, by using several such loops to form a receive coil, we can measure a signal of the form:

$$
S_{0}(t)=\frac{\chi \omega_{0}^{2} e^{i \omega_{0} t}}{\gamma} \int_{\text {sample }} \rho(x, y, z) e^{-\frac{t}{T_{2}(x, y, z)}} b_{1 \mathrm{rec}}(x, y, z) d x d y d z .
$$

Here $b_{1 \text { rec }}(x, y, z)$ quantifies the sensitivity of the receive coil to the precessing magnetization located at $(x, y, z)$. From $S_{0}(t)$ we easily obtain a measurement of the integral of the function $e^{-\frac{t}{T_{2}}} \rho b_{1 \mathrm{rec}}$. By using a carefully designed receive coil, $b_{1 \text { rec }}$ can be taken to be a constant, and therefore we can determine the total spin density, weighted by $e^{-\frac{t}{T_{2}}}$, within the object of interest. For the rest of this article we assume that $b_{1 \text { rec }}$ is a constant. Note that the size of the measured signal is proportional to $\omega_{0}^{2}$, which is, in turn, proportional to $\left\|\boldsymbol{B}_{0}\right\|^{2}$. This explains, in part, why it is so useful to have a very 
strong $\boldsymbol{B}_{0}$-field. Though even with a 1.5 Tesla magnet, the measured signal is only in the micro-watt range, see $[14,7]$.

Suppose that at the end of the RF-excitation, we turn on the gradient $\tilde{\boldsymbol{G}}$. As the magnetic field $\boldsymbol{B}=\boldsymbol{B}_{0}+\widetilde{\boldsymbol{G}}$ now has a nontrivial spatial dependence, the precessional frequency of the magnetization, which equals $\gamma\|\boldsymbol{B}\|$, also has a spatial dependence. In fact, assuming that $T_{2}$ is spatially independent, it follows from (11), that the measured signal would now be given by

$$
S_{\boldsymbol{G}}(t) \approx \frac{\chi b_{1 \mathrm{rec}} \omega_{0}^{2} e^{-\frac{t}{T_{2}}} e^{i \omega_{0} t}}{\gamma} \int_{\text {sample }} \rho(x, y, z) e^{2 \pi i\langle(x, y, z), \boldsymbol{k}\rangle} d x d y d z .
$$

Up to a constant, $e^{-i \omega_{0} t} e^{-\frac{t}{T_{2}}} S_{\boldsymbol{G}}(t)$ is simply the Fourier transform of $\rho$ at $\boldsymbol{k}=-\frac{t \gamma \boldsymbol{G}}{2 \pi}$. By sampling in time and using a variety of different gradient vectors, we can sample the $3 \mathrm{~d}$ Fourier transform of $\rho$ in a neighborhood of 0 . This suffices to reconstruct an approximation to $\rho$. In medical applications $T_{2}$ is spatially dependent, which, as described in Section 7, provides a useful contrast mechanism.

Imagine that we collect samples of $\hat{\rho}(\boldsymbol{k})$ on a rectangular grid

$$
\left\{\left(j_{x} \Delta k_{x}, j_{y} \Delta k_{y}, j_{z} \Delta k_{z}\right):-\frac{N_{x}}{2} \leq j_{x} \leq \frac{N_{x}}{2},-\frac{N_{y}}{2} \leq j_{y} \leq \frac{N_{y}}{2},-\frac{N_{z}}{2} \leq j_{z} \leq \frac{N_{z}}{2}\right\} .
$$

Since we are sampling in the Fourier domain the Nyquist sampling theorem implies that the sample spacing determines the spatial field-of-view from which we can reconstruct an artifact free image: in order to avoid aliasing artifacts, the support of $\rho$ must lie in a rectangular region with side lengths $\left[\Delta k_{x}^{-1}, \Delta k_{y}^{-1}, \Delta k_{z}^{-1}\right]$, see $[13,10,2]$. In typical medical applications the support of $\rho$ is much larger in one dimension than the others and so it turns out to be impractical to use the simple data collection technique described above. Instead the RF-excitation takes place in the presence of non-trivial gradient fields, which allows for a spatially selective excitation: the magnetization in one region of space obtains a transverse component, while that in the complementary region is left in the equilibrium state. In this way we can collect data from an essentially two dimensional slice. This is described in the next section.

\section{Selective excitation}

As remarked above, practical imaging techniques do not excite all the spins in an object and directly measure samples of the $3 \mathrm{~d}$ Fourier transform. Rather the spins lying in a slice are excited and samples of the $2 \mathrm{~d}$ Fourier transform are then measured. This process is called selective excitation and may be accomplished by applying the RF-excitation with a gradient field turned on. With this arrangement, the strength of the static field, $\boldsymbol{B}_{0}+\widetilde{\boldsymbol{G}}$, varies with spatial position, hence the response to the RF-excitation does as well. Suppose that $\widetilde{\boldsymbol{G}}=(0,0,\langle(x, y, z), \boldsymbol{G}\rangle)$ and set $f=[2 \pi]^{-1} \gamma\langle(x, y, z), \boldsymbol{G}\rangle$. This is called the offset frequency as it is the amount by which the local resonance frequency differs from the resonance frequency, $\omega_{0}$ of the 
uniform $\boldsymbol{B}_{0}$-field. The result of a selective RF-excitation is described by a magnetization profile $\boldsymbol{m}^{\mathrm{pr}}(f)$, this is a unit 3-vector valued function of the offset frequency. A typical case would be

$$
\boldsymbol{m}^{\mathrm{pr}}(f)= \begin{cases}{[0,0,1]} & \text { for } f \notin\left(f_{0}, f_{1}\right) \\ {[\sin \theta, 0, \cos \theta]} & \text { for } f \in\left(f_{0}, f_{1}\right)\end{cases}
$$

The magnetization is flipped through an angle $\theta$, in regions of space where the offset frequency lies in $\left(f_{0}, f_{1}\right)$ and is left in the equilibrium state otherwise.

Typically the excitation step takes a few milliseconds and is much shorter than either $T_{1}$ or $T_{2}$; therefore one generally uses the Bloch equation, without relaxation, in the discussion of selective excitation. In the rotating reference frame the Bloch equation, without relaxation, takes the form

$$
\frac{d \boldsymbol{m}(f ; t)}{d t}=\left[\begin{array}{ccc}
0 & 2 \pi f & -\gamma \beta \\
-2 \pi f & 0 & \gamma \alpha \\
\gamma \beta & -\gamma \alpha & 0
\end{array}\right] \boldsymbol{m}(f ; t) .
$$

The problem of designing a selective pulse is non-linear. Indeed, the selective excitation problem can be rephrased as a classical inverse scattering problem: one seeks a function $\alpha(t)+i \beta(t)$ with support in an interval $\left[t_{0}, t_{1}\right]$ so that, if $\boldsymbol{m}(f ; t)$ is the solution to (17) with $\boldsymbol{m}\left(f ; t_{0}\right)=[0,0,1]$, then $\boldsymbol{m}\left(f ; t_{1}\right)=\boldsymbol{m}^{\mathrm{pr}}(f)$. If one restricts attention to flip angles close to 0 , then there is a simple linear model that can be used to find approximate solutions.

If the flip angle is close to zero, then $m_{3} \approx 1$ throughout the excitation. Using this approximation, we derive the low flip angle approximation to the Bloch equation, without relaxation:

$$
\frac{d\left(m_{1}+i m_{2}\right)}{d t}=-2 \pi i f\left(m_{1}+i m_{2}\right)+i \gamma(\alpha+i \beta) .
$$

From this approximation we see that

$$
\alpha(t)+i \beta(t) \approx \frac{\mathscr{F}\left(m_{1}^{\mathrm{pr}}+i m_{2}^{\mathrm{pr}}\right)(t)}{\gamma i}, \text { where } \mathscr{F}(h)(t)=\int_{-\infty}^{\infty} h(f) e^{-2 \pi i f t} d f .
$$

For an example like that in (16), $\theta$ close to zero and $f_{0}=-f_{1}$, we obtain

$$
\alpha+i \beta \approx \frac{i \sin \theta \sin f_{1} t}{\pi \gamma t} .
$$

A pulse of this sort is called a sinc-pulse. A sinc-pulse is shown in Figure 1(a), the result of applying it in Figure 1(b). A more accurate pulse can be designed using the Shinnar-Le Roux algorithm, see [16, 17], or the inverse scattering approach, see [11]. An inverse scattering $90^{\circ}$-pulse is shown in Figure 2(a) and the response in Figure 2(b). 


\section{Spin-warp imaging}

In Section 4 we showed how NMR measurements could be used to measure the $3 \mathrm{~d}$ Fourier transform of $\rho$. In this section we consider a more practical technique, that of measuring the $2 \mathrm{~d}$ Fourier transform of a "slice" of $\rho$. Applying a selective RF-pulse, as described in the previous section, we can flip the magnetization in a region of space $z_{0}-\Delta z<z<z_{0}+\Delta z$, while leaving it in the equilibrium state outside a slightly larger region. Observing that a signal near the resonance frequency is only produced by isochromats whose magnetization has a non-zero transverse component, we can now measure samples of the $2 \mathrm{~d}$ Fourier transform of the function

$$
\bar{\rho}_{z_{0}}(x, y)=\frac{1}{2 \Delta z} \int_{z_{0}-\Delta z}^{z_{0}+\Delta z_{0}} \rho(x, y, z) d z .
$$

If $\Delta z$ is sufficiently small then $\bar{\rho}_{z_{0}}(x, y) \approx \rho\left(x, y, z_{0}\right)$.

In order to be able to use the fast Fourier transform algorithm (FFT) to do the reconstruction, it is very useful to sample $\widehat{\bar{\rho}}_{z_{0}}$ on a uniform grid. To that end we use the gradient fields as follows: After the RF-excitation we apply a gradient field of the form $\boldsymbol{G}_{\mathrm{ph}}=\left(0,0,-g_{2} y+g_{1} x\right)$, for a certain period of time, $T_{\mathrm{ph}}$. This is called a phase encoding gradient. At the conclusion of the phase encoding gradient, the transverse components of the magnetization from the excited spins has the form

$$
\boldsymbol{m}^{\|}(x, y) \propto\left[\cos 2 \pi\left(k_{y} y-k_{x} x\right),-\sin 2 \pi\left(k_{y} y-k_{x} x\right)\right] \bar{\rho}_{z_{0}}(x, y),
$$

where $\left(k_{x}, k_{y}\right)=[2 \pi]^{-1} \gamma T_{\mathrm{ph}}\left(-g_{1}, g_{2}\right)$. At time $T_{\mathrm{ph}}$ we turn off the $y$-component of $\boldsymbol{G}_{\mathrm{ph}}$ and reverse the polarity of the $x$-component. At this point we begin to measure the signal. We get samples of $\widehat{\bar{\rho}}\left(k, k_{y}\right)$ where $k$ varies from $-k_{x}$ max to $k_{x} \max$. By repeating this process with the strength of the $y$-phase encoding gradient being stepped through a sequence of uniformly spaced values, $g_{2} \in\left\{n \Delta g_{y}\right\}$, and collecting samples at a uniformly spaced set of times, we collect the set of samples

$$
\left\{\widehat{\bar{\rho}}_{z_{0}}\left(m \Delta k_{x}, n \Delta k_{y}\right):-\frac{N_{x}}{2} \leq m \leq \frac{N_{x}}{2},-\frac{N_{y}}{2} \leq n \leq \frac{N_{y}}{2}\right\} .
$$

The gradient $\boldsymbol{G}_{\mathrm{fr}}=\left(0,0,-g_{1} x\right)$, left on during signal acquisition, is called a frequency encoding gradient. While there is no difference mathematically between the phase encoding and frequency encoding steps, there are significant practical differences. This approach to sampling is known as spin-warp imaging; it was introduced in [8]. The steps of this experiment are summarized in a pulse sequence timing diagram, shown in Figure 3. This graphical representation for the steps followed in a magnetic resonance imaging experiment is ubiquitous in the literature.

To avoid aliasing artifacts, the sample spacings $\Delta k_{x}$ and $\Delta k_{y}$ must be chosen so that the excited portion of the sample is contained in a region of size $\Delta k_{x}^{-1} \times \Delta k_{y}^{-1}$. This is called the field-of-view or FOV. Since we can only collect the signal for a finite period of time, the Fourier transform $\widehat{\bar{\rho}}\left(k_{x}, k_{y}\right)$ is sampled at frequencies lying in a rectangle with vertices $\left( \pm k_{x \max }, \pm k_{y \text { max }}\right)$, where

$$
k_{x \max }=\frac{N_{x} \Delta k_{x}}{2}, \quad k_{y \max }=\frac{N_{y} \Delta k_{y}}{2} .
$$


The maximum frequencies sampled effectively determine the resolution available in the reconstructed image. Heuristically, this resolution limit equals half the shortest measured wavelength:

$$
\Delta x \approx \frac{1}{2 k_{x \max }}=\frac{F O V_{x}}{N_{x}} \quad \Delta y \approx \frac{1}{2 k_{y \max }}=\frac{F O V_{y}}{N_{y}} .
$$

Whether one can actually resolve objects of this size in the reconstructed image depends on other factors such as the available contrast and the signal-to-noise ratio (SNR). We consider these factors in the final sections.

\section{Signal-to-Noise}

At a given spatial resolution, image quality is largely determined by the signal-to-noise ratio (SNR) and contrast between the different materials making up the imaging object. SNR in MRI is defined as the voxel signal amplitude divided by the noise standard deviation. The noise in the NMR signal, in general, is Gaussian distributed with zero mean. Ignoring contributions from quantization, for example, due to limitations of the analog-to-digital converter, the noise voltage of the signal can be ascribed to random thermal fluctuations in the receive circuit, see [7]. The variance is given by

$$
\sigma_{\text {thermal }}^{2}=4 k_{B} T R \Delta v,
$$

where $k_{B}$ is Boltzmann's constant, $T$ is the absolute temperature, $R$ is the effective resistance (resulting from both receive coil, $R_{c}$ and object, $R_{o}$ ), and $\Delta v$ is the receive bandwidth. Both $R_{c}$ and $R_{o}$ are frequency dependent, with $R_{c} \propto \omega^{\frac{1}{2}}$, and $R_{o} \propto$ $\omega$. Their relative contributions to overall circuit resistance depend in a complicated manner on coil geometry, and the imaging object's shape, size and conductivity, see [6]. Hence, at high magnetic field, and for large objects, as in most medical applications, the resistance from the object dominates and the noise scales linearly with frequency. Since the signal is proportional to $\omega^{2}$, in MRI, the SNR increases in proportion to the field strength.

As the reconstructed image is complex valued, it is customary to display the magnitude rather than the real component. Doing so, however, has some consequences on the noise properties. In regions where the signal is much larger than the noise, the Gaussian approximation is valid. However, in regions where the signal is low, rectification causes the noise to assume a Raleigh distribution. Mean and standard deviation can be calculated from the joint probability distribution:

$$
P\left(N_{r}, N_{i}\right)=\frac{1}{2 \pi \sigma^{2}} e^{-\left(N_{r}^{2}+N_{i}^{2}\right) / 2 \sigma^{2}},
$$

where $N_{r}$ and $N_{i}$ are the noise in the real and imaginary channels. When the signal is large compared to noise, one finds that the variance $\sigma_{m}^{2}=\sigma^{2}$. In the other extreme of nearly zero signal, one obtains for the mean:

$$
\widehat{S}=\sigma \sqrt{\pi / 2} \cong 1.253 \sigma
$$


and, for the variance:

$$
\sigma_{m}^{2}=2 \sigma^{2}(1-\pi / 4) \cong 0.655 \sigma^{2}
$$

Of particular practical significance is the SNR dependence on the imaging parameters. The voxel noise variance is reduced by the total number of samples collected during the data acquisition process, i.e.

$$
\sigma_{m}^{2}=\sigma_{\text {thermal }}^{2} / N,
$$

where $N=N_{x} N_{y}$ in a 2d spin-warp experiment. Incorporating the contributions to thermal noise variance, other than bandwidth, into a constant

$$
u=4 k_{B} T R,
$$

we obtain for the noise variance:

$$
\sigma_{m}^{2}=\frac{u \Delta v}{N_{x} N_{y} N_{a v g}} .
$$

Here $N_{a v g}$ is the number of signal averages collected at each phase-encoding step. We obtain a simple formula for SNR per voxel of volume $\Delta V$ :

$$
S N R=C \tilde{\rho} \Delta V \sqrt{\frac{N_{x} N_{y} N_{a v g}}{u \Delta v}}=C \tilde{\rho} \Delta x \Delta y d_{z} \sqrt{\frac{N_{x} N_{y} N_{a v g}}{u \Delta v}},
$$

where $\Delta x, \Delta y$ are defined in (25), $d_{z}$ is the thickness of the slab selected by the sliceselective RF pulse, and $\tilde{\rho}$ denotes the spin density weighted by effects determined by the (spatially varying) relaxation times $T_{1}$ and $T_{2}$ and the pulse sequence timing parameters. Figure 4 shows two images of the human brain obtained from the same anatomic location but differing in SNR.

\section{Contrast and Resolution}

The single most distinctive feature of MRI is its extraordinarily large innate contrast. For two soft tissues, it can be on the order of several hundred percent. By comparison, contrast in X-ray imaging is a consequence of differences in the attenuation coefficients for two adjacent structures and is typically on the order of a few percent.

We have seen in the preceding sections that the physical principles underlying MRI are radically different from those of X-ray computed tomography in that the signal elicited is generated by the spins themselves in response to an external perturbation. The contrast between two regions, $A$ and $B$, with signals $S_{A}, S_{B}$ respectively, is defined as

$$
C_{A B}=\frac{S_{A}-S_{B}}{S_{A}} .
$$

If the only contrast mechanism were differences in the proton spin density of various tissues, then contrast would be on the order of 5-20\%. In reality, it can be several hundred percent. The reason for this discrepancy is that the MR signal is acquired under non-equilibrium conditions. At the time of excitation, the spins have typically 
not recovered from the effect of the previous cycle's RF pulses, nor is the signal usually detected immediately after its creation.

Typically, in spin-warp imaging, a spin-echo is detected as a means to alleviate spin coherence losses from $\boldsymbol{B}_{0}$-field inhomogeneity. A spin echo is the result of applying an RF-pulse that has the effect of taking $\left(m_{1}, m_{2}, m_{3}\right)$ to $\left(m_{1},-m_{2},-m_{3}\right)$. As such a pulse effects a $180^{\circ}$ rotation of the $\hat{z}$-axis, it is also called a $\pi$-pulse. If, after a $\pi$-pulse, the spins continue to evolve in the same environment then, following a certain period of time, the transverse components of the magnetization vectors throughout the sample become aligned. Hence a pulse of this type is also called a refocusing pulse. The time when all the transverse components are refocused is called the echo time, TE.

The spin-echo signal amplitude for an RF pulse sequence $\frac{\pi}{2}-\tau-\pi-\tau$, repeated every $T_{R}$, seconds is approximately given by:

$$
S(t=2 \tau) \approx \rho\left(1-e^{-T_{R} / T_{1}}\right) e^{-T E / T_{2}} .
$$

This is a good approximation as long as $T E<<T_{R}$ and $T_{2}<<T_{R}$ in which case the transverse magnetization decays essentially to zero between successive pulse sequence cycles. In equation (35), $\rho$ is voxel spin density and the echo time $T E=2 \tau$. Empirically, it is known that tissues differ in at least one of the intrinsic quantities, $T_{1}, T_{2}$ or $\rho$. It therefore suffices to acquire images in such a manner that contrast is sensitive to one particular parameter. For example, a " $T_{2}$-weighted" image would be acquired with $T E \sim T_{2}$ and $T R>>T_{1}$ and, similarly, a " $T_{1}$-weighted" image with $T R<T_{1}$ and $T E<<T_{2}$, with $T_{1}, T_{2}$ representing typical tissue proton relaxation times. Figure 5 shows two images obtained with the same scan parameters except for $T R$ and $T E$ illustrating the fundamentally different image contrasts that are achievable.

It is noteworthy that object visibility is not just determined by the contrast between adjacent structures but is also a function of the noise. It is therefore useful to define the contrast-to-noise ratio as

$$
C N R_{A B}=\frac{C_{A B}}{\sigma_{\mathrm{eff}}}
$$

where $\sigma_{\text {eff }}$ is the effective standard deviation of the signal. Finally, it may be useful to reconstruct parametric images in which the pixel signal values represent any one of the intrinsic parameters. A $T_{2}$-image can be computed from equation (35), for example, either analytically from two image data sets acquired with two different echo times, or from a series of $T E$ values, obtained from a Carr-Purcell spin-echo train, using regression techniques, see $[5,13]$.

We have previously shown that the limiting resolution is given by $k_{\max }$, the largest spatial frequency sampled, see (25). In reality, however, the actual resolution is always lower. For example spin-spin $\left(T_{2}\right)$ relaxation causes the signal to decay during the acquisition. In spin-warp imaging this causes the high spatial frequencies to be further attenuated.

A further consequence of finite sampling is a ringing or Gibbs artifact that is most prominent at sharp intensity discontinuities. In practice, these artifacts are mitigated by applying an appropriate apodizing filter to the data. Figure 6 shows a portion of a brain image obtained at two different resolutions. In Figure 6(b), the total $\boldsymbol{k}$-space area covered was 16 times larger than for the acquisition of the image in b). Artifacts from 
finite sampling and blurring of fine detail such cortical blood vessels are clearly visible in the low-resolution image. SNR, according to equation (33), is reduced in the latter image by a factor of 4 .

\section{References}

[1] A. Abragam, Principles of Nuclear Magnetism, Clarendon Press, Oxford, 1983.

[2] H. H. BARRetT AND K. J. MYers, Foundations of Image Science, John Wiley and Sons, Hoboken, 2004.

[3] M. A. Bernstein, K. F. King, And X. J. Zhou, Handbook of MRI Pulse Sequences, Elsevier Academic Press, London, 2004.

[4] P. T. Callaghan, Principles of Nuclear Magnetic Resonance Microscopy, Clarendon Press, Oxford, 1993.

[5] P. T. Callaghan, 438 Nuclear Magnetic Resonance, Encyclopedia of Mathematics and Physics, Elsevier, 2006.

[6] C.-N. Chen And D. I. Hoult, Biomedical Magnetic Resonance Technology, Adam Hilger, Bristol, 1989.

[7] W. A. Edelstein, G. H. Glover, C. Hardy, and R. Redington, The intrinsic signal-to-noise ratio in NMR imaging, Magn. Reson. Med., 3 (1986), pp. 604-618.

[8] W. A. Edelstein, J. M. Hutchinson, J. M. Johnson, and T. Redpath, Spin warp NMR imaging and applications to human whole-body imaging, Phys. Med. Biol., 25 (1980), pp. 751-756.

[9] Nick Woodhouse, Introductory articles: Electromagnetism, Encyclopedia of Mathematical Physics, Elsevier, 2006.

[10] C. L. Epstein, Introduction to the Mathematics of Medical Imaging, Prentice Hall, Upper Saddle River, NJ, 2003.

[11] - Minimum power pulse synthesis via the inverse scattering transform, Jour. Mag. Res., 167 (2004), pp. 185-210.

[12] R. ERnst, G. Bodenhausen, and A. Wokaun, Principles of Nuclear Magnetic Resonance in One and Two Dimensions, Clarendon, Oxford, 1987.

[13] E. M. HaAcke, R. W. Brown, M. R. Thompson, and R. Venkatesan, Magnetic Resonance Imaging, Wiley-Liss, New York, 1999.

[14] D. Hoult AND P. C. LAuterbur, The sensitivity of the zeugmatographic experiment involving human samples, JMR, 34 (1979), pp. 425-433. 
[15] M. H. LEvitT, Spin Dynamics, Basics of Nuclear Magnetic Resonance, John Wilea \& Sons, Chichester, 2001.

[16] J. Pauly, P. Le Roux, D. Nishimura, And A. Macovski, Parameter relations for the Shinnar-Le Roux selective excitation pulse design algorithm, IEEE Trans. on Med. Imaging, 10 (1991), pp. 53-65.

[17] M. SHINNAR AND J. LEIGH, The application of spinors to pulse synthesis and analysis, Mag. Res. in Med., 12 (1989), pp. 93-98.

[18] C. P. Slichter, Principles of Magnetic Resonance,3rd enl. and upd. ed., Springer series in solid-state sciences, 1, Springer-Verlag, Berlin, New York, 1990.

[19] F. W. WEHRLI, From NMR diffraction and zeugmatography to modern imaging and beyond, Progress in Nuclear Magnetic Resonance Spectroscopy, Eds. J. W. Emsley, J. Feeney and L. H. Sutcliffe, 28 (1995), pp. 87-135.

[20] Nick WoOdhouse, 387 Introductory articles: Electromagnetism, Encyclopedia of Mathematical Physics, Elsevier, 2006.

\section{Figures}

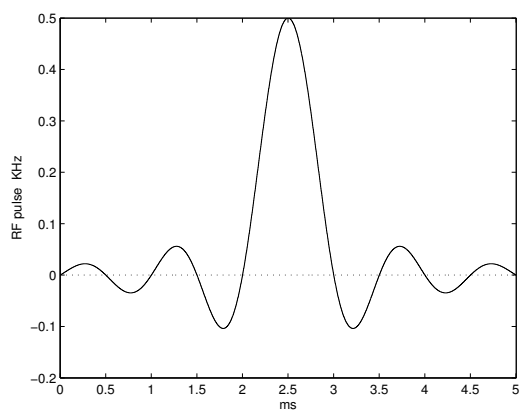

(a) Profile of a $90^{\circ}$-sinc pulse

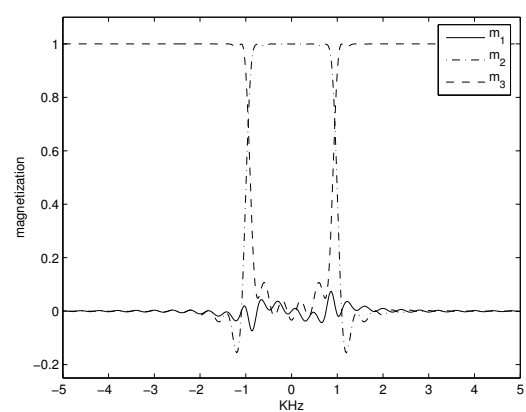

(b) The magnetization profile produced by the pulse in (a).

Figure 1. A selective $90^{\circ}$-pulse and profile designed using the linear approximation. 


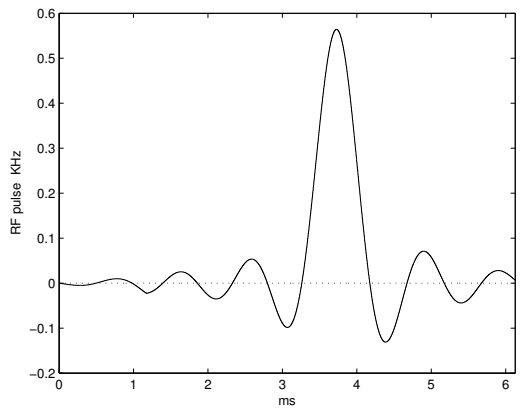

(a) Profile of a $90^{\circ}$ inverse scattering pulse.

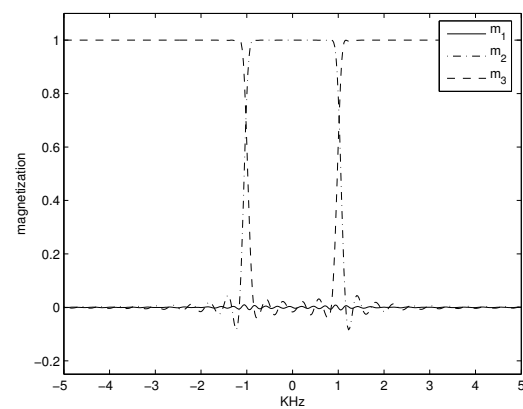

(b) The magnetization profile produced by the pulse in (a).

Figure 2. A selective $90^{\circ}$-pulse and profile designed using the inverse scattering approach.

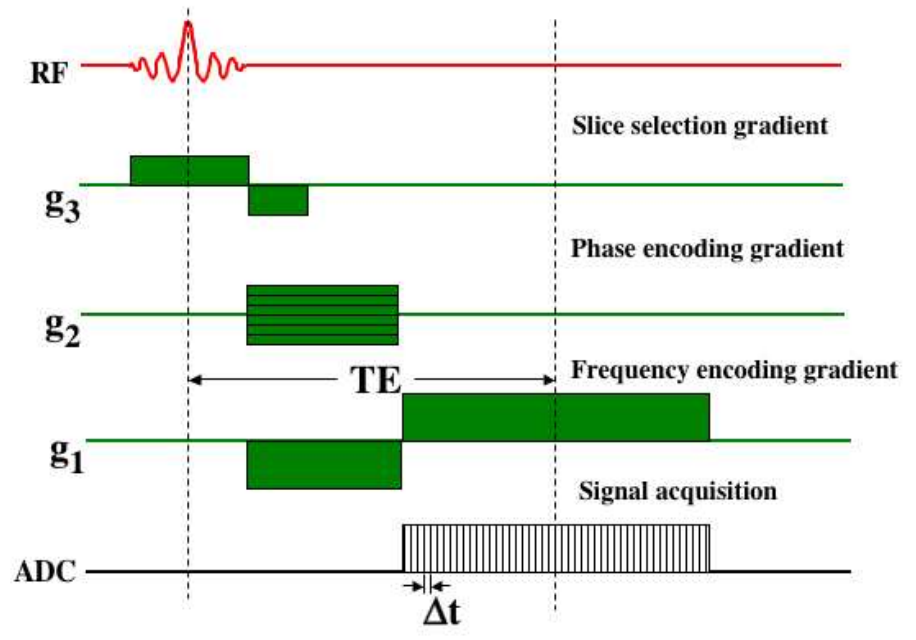

Figure 3. Pulse timing diagram for spin-warp imaging. During the positive lobe of the frequency-encoding gradient, the analog-to-digital converter (ADC) collects samples of the signal produced by the rotating transverse magnetization. 


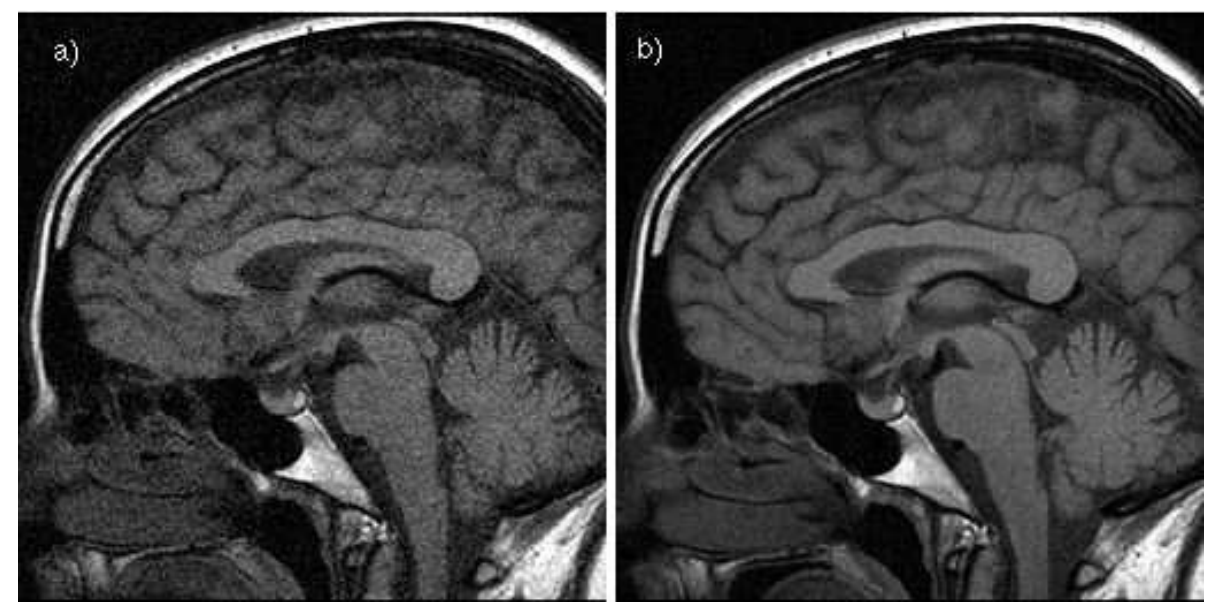

Figure 4. $\mathrm{T}_{1}$-weighted sagittal images through the midline of the brain: Image $\mathrm{b}$ ) has twice the SNR of image a) showing improved conspicuity of small anatomic and low-contrast detail. The two images were acquired at 1.5 Tesla field strength using $2 \mathrm{D}$ spin-warp acquisition and identical scan parameters, except for $N_{\text {avg }}$, which was 1 in a) and 4 in b).

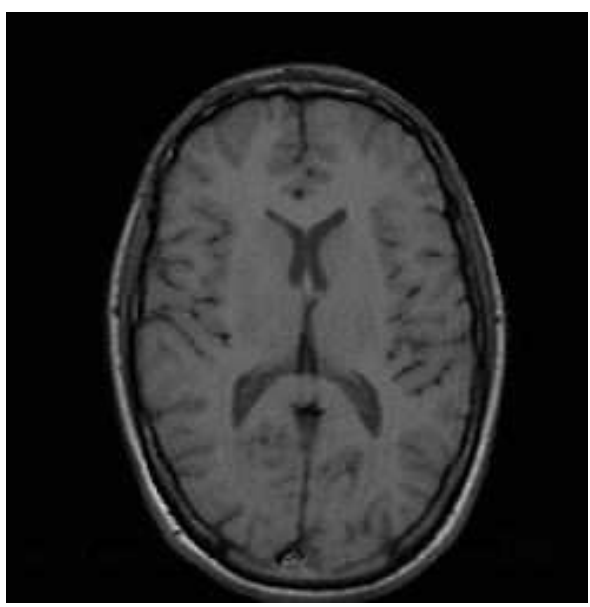

(a)

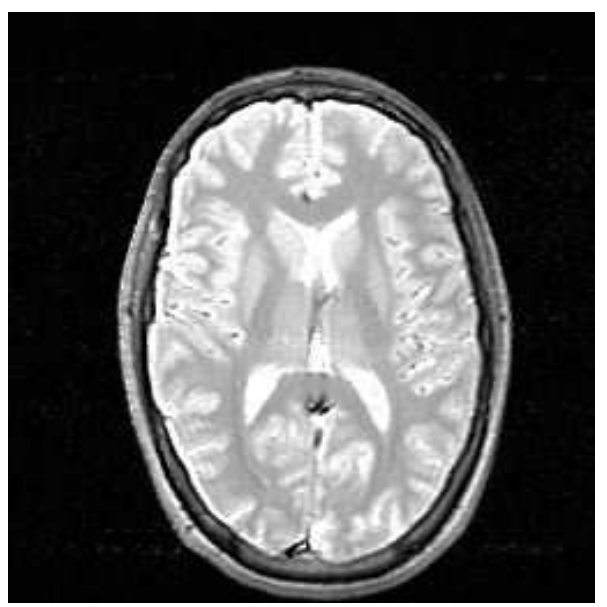

(b)

Figure 5. Dependence of image contrast on pulse sequence timing parameters: a) is a $T_{1}$-weighted image; b) is a proton density-weighted image. 


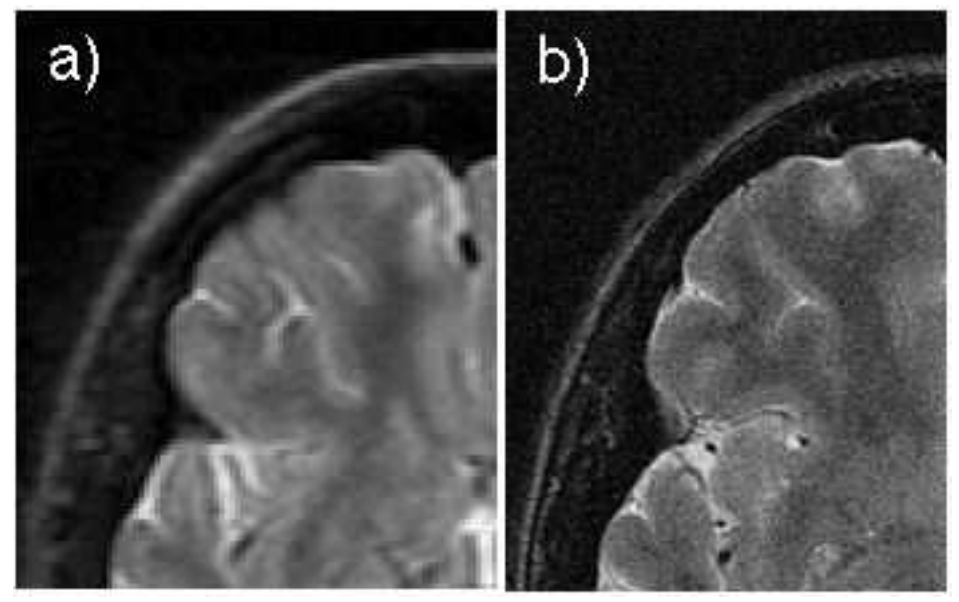

Figure 6. Effect of $\boldsymbol{k}$-space coverage on spatial resolution in axial image of the brain: the field of view in both images $20 \mathrm{~cm}$ and all scan parameters were the same except that a) was acquired with $N_{x}=N_{y}=128$ and $N_{x}=N_{y}=512$. 